\title{
EFEITOS DO TREINAMENTO DE FORÇA NA FLEXIBILIDADE EM IDOSOS: REVISÃO DE LITERATURA
}

\section{ARTIGO DE REVISÃo}

CARVALHO, Murilo Alves ${ }^{1}$, CANTÃO, Elbert Wander ${ }^{2}$

CARVALHO, Murilo Alves. CANTÃO, Elbert Wander. Efeitos do treinamento de força na flexibilidade em idosos: revisão de literatura. Revista Científica Multidisciplinar Núcleo do Conhecimento. Ano. 07, Ed. 02, Vol. 02, pp. 139-156. Fevereiro de 2022. ISSN: 2448-0959, Link de acesso: https://www.nucleodoconhecimento.com.br/educacao-fisica/efeitos-dotreinamento

\section{RESUMO}

A população idosa tem aumentado consideravelmente em todo o mundo. $O$ envelhecimento é um processo inevitável, variando somente de indivíduo para indivíduo. No processo do envelhecimento são notados diversos declínios físicos como a diminuição da flexibilidade, força e massa muscular, tornando os idosos mais frágeis, com limitação funcional e dificultando a realização de suas atividades diárias. Sabe-se que o treinamento de força na terceira idade visa proporcionar o enfrentamento desse novo modo de vida com menos limitações, mais independência, resgatando a sua autoestima que é um fator determinante em relação a manutenção da qualidade de vida e do bem-estar desta população. A flexibilidade em indivíduos idosos é um componente importante da aptidão física e a sua boa manutenção garante uma maior amplitude nos movimentos corporais, promovendo melhorias na execução de atividades cotidianas com efeitos benéficos na qualidade de vida. Sendo assim, seria o treinamento de força em idosos capaz de melhorar, além da força, a

${ }^{1}$ Graduação em Educação Física. ORCID: 0000-0002-0187-7715

2 Orientador. ORCID: 0000-0003-3756-7652

RC: 106653

Disponível em: https://www.nucleodoconhecimento.com.br/educacao-fisica/efeitosdo-treinamento 
flexibilidade desse público? O presente estudo, do tipo descritivo e bibliográfico, teve como objetivo geral buscar a interação entre as capacidades físicas força e flexibilidade no treinamento de força em idosos e forma mais específica, conhecer quais são os efeitos do treinamento de força na flexibilidade dos idosos. Para a coleta dos dados, foram utilizadas as bases científicas eletrônicas: PubMed, SciELO, Google Acadêmico dentre outras. Ao final, 39 trabalhos foram selecionados para o desenvolvimento desse trabalho. Segundo a literatura revisada, conclui-se que o treinamento de força em idosos proporcionou diversos benefícios, como o aumento de força, resistência e também foi capaz de melhorar a flexibilidade do público-alvo. Todos esses benefícios geraram um ganho no desempenho funcional e melhora da qualidade vida, retardando alguns efeitos fisiológicos do envelhecimento. Dessa forma, cabe aos profissionais de educação física utilizar os conhecimentos adquiridos, tanto teóricos como práticos, como forma de prescrever a atividade de maneira correta e segura, possibilitando o idoso envelhecer com saúde e melhor qualidade de vida.

Palavras-chave: Treinamento de força, Flexibilidade, Idosos, Envelhecimento, Qualidade de vida.

\section{INTRODUÇÃO}

Observa-se que a população idosa tem aumentado consideravelmente no mundo e esse fato tem despertado a atenção de diversas áreas do conhecimento a fim de elaborar políticas públicas direcionadas para o envelhecimento saudável dessa população. Sabe-se que o envelhecimento é um processo que ocorre de maneira natural em todos seres vivos, é um processo inevitável, variando somente de indivíduo para indivíduo. (CIOSAK, 2011; ALBINO, 2012; SILVA, 2017)

De acordo com Silva (2017) o idoso é aquele indivíduo com mais de 60 anos em países em desenvolvimento e 65 anos em nações desenvolvidas. Estes parâmetros de medição são critérios utilizados pela maioria das instituições que buscam dar aos idosos atenção à saúde física, psicológica e social. Já Shephard (2003) classifica os indivíduos idosos situando-os em categorias funcionais: velhice; velhice avançada; e

RC: 106653

Disponível em: https://www.nucleodoconhecimento.com.br/educacao-fisica/efeitosdo-treinamento 
velhice muito avançada. A velhice compreende o intervalo entre 65 e 75 anos. A velhice avançada é a categoria etária que compreende a faixa situada entre 75 e 85 anos, e a velhice muito avançada compreende a faixa etária acima dos 85 anos.

Com o desenvolvimento da medicina, a expectativa de vida tende a aumentar e, consequentemente, pacientes com idade mais avançada têm procurado os cuidados profissionais da saúde, motivo que leva a um estudo mais aprofundado da senilidade (CIOSAK et al., 2011). O Brasil iniciou o novo século com a população idosa crescendo proporcionalmente quase oito vezes mais que os jovens e quase duas vezes mais que a população em geral, segundo dados do Instituto Brasileiro de Geografia e Estatística (MIRANDA; MENDES; SILVA, 2016).

Albino et al. (2012) define o treinamento de força como um método de treinamento que utiliza o levantamento de pesos. Amplamente conhecido e utilizado, é capaz de aumentar a força e a massa muscular em diferentes públicos como atletas, indivíduos ativos fisicamente e idosos que também buscam essas adaptações.

Ainda, segundo Dantas (2005), os idosos podem beneficiar-se do treinamento de força necessitando de maior acompanhamento de um profissional habilitado para a orientação e monitoramento das atividades, minimizando os riscos e ressaltando os benefícios, que não são poucos e serão detalhados posteriormente.

Já a flexibilidade, incide na capacidade que um indivíduo tem de executar movimentos de grande amplitude pelas forças extremas ou ainda que requeiram a movimentação de muitas articulações, como mobilidade, elasticidade, capacidade de articulação (WEINECK, 2003).

De acordo com Gonçalves; Gurjão e Gobbi (2007) a flexibilidade é determinada como a capacidade de movimento da articulação com a máxima amplitude possível, declinando de $20 \%$ a $30 \%$ dos 20 aos 70 anos, tendo um aumento nessa percentagem depois dos 80 anos. 
Essas duas capacidades físicas possuem relação direta com a qualidade de vida e autonomia do idoso (MATSUDO, 2010; SILVA, 2017; LOPES, 2018). Mas seria o treinamento de força em idosos capaz de melhorar, além da força, a flexibilidade desse público?

Sendo assim, o presente estudo, do tipo descritivo e bibliográfico, teve como objetivo geral, buscar a interação entre as capacidades físicas força e flexibilidade, de forma mais específica, conhecer quais são os efeitos do treinamento de força na flexibilidade dos idosos, e verificar se esses efeitos serão positivos ou não na vida diária do idoso.

Para a coleta de dados foram utilizadas as bases científicas eletrônicas: PubMed, SciELO, Google Acadêmico dentre outras. Foram selecionados artigos nessas bases utilizando as seguintes palavras-chave: treinamento de força, flexibilidade, idosos, envelhecimento e qualidade de vida. Além disso, foram utilizados livros de fisiologia humana e do exercício para a elaboração deste trabalho. A seleção foi realizada entre os meses de Abril a Agosto de 2020. Por se tratar de termos de busca amplos, vários artigos foram encontrados. Como critérios de inclusão, foram selecionados artigos e livros de 1998 até 2020 . Foram descartados artigos fora do período citado e aqueles que não relacionavam de forma direta pelo menos duas das palavras-chave. Ao final, 39 trabalhos foram selecionados para o desenvolvimento desse trabalho.

\section{O IDOSO E O PROCESSO DE ENVELHECIMENTO}

Os seres humanos passam por um ciclo vital, os quais nascem, crescem, amadurecem, envelhecem e morrem (JARDIM, 2007). Como parte deste ciclo, o processo de envelhecimento é essencial a todos os seres vivos, de forma que quando um indivíduo chega à velhice é considerado uma conquista daqueles que ultrapassaram os desafios da vida (JARDIM, 2007; SILVA, 2017).

O processo de envelhecimento é acompanhado, muitas vezes, por um estilo de vida inativo. Esse fator favorece o declínio das capacidades físicas, o que gera perdas na aptidão funcional e consequente dependência. Grandes modificações no organismo

RC: 106653

Disponível em: https://www.nucleodoconhecimento.com.br/educacao-fisica/efeitosdo-treinamento 
dos idosos são vistas e podem ser observadas em todos os aparelhos e sistemas: muscular, ósseo, circulatório, pulmonar, endócrino e imunológico (NASCIMENTO et al., 2013; MENDONÇA; MOURA; LOPES, 2018).

Nesse processo, os idosos tornam-se mais frágeis e com maior dificuldade na realização das atividades diárias devido a diminuição da força e massa muscular. Pode-se observar ainda que, com o treinamento de força, os idosos poderão adquirir aumento de força, resistência e flexibilidade, o que gera um ganho no desempenho funcional, melhora a qualidade de vida, retardo de alguns efeitos fisiológicos do envelhecimento (MATSUDO, 2010).

Conforme esclarece Locks et al. (2012), com o envelhecimento, existe perda de massa muscular com diminuição do peso, da área de secção transversal e do número de células. O nível de atividade física, seu estado nutricional e aspectos hereditários influenciam a perda de células musculares nessa fase da vida. Já o sistema nervoso é o sistema fisiológico mais comprometido com o processo do envelhecimento. Ele é responsável por diferentes tipos de sensações, movimentos, funções psíquicas, dentre outros. As alterações mais importantes, características do envelhecimento, ocorrem no cérebro.

O equilíbrio, a postura, a velocidade da marcha, o aumento no risco de quedas, a dificuldade de levantar de uma cadeira e a dificuldade em realizar tarefas diárias estão associadas a perda de força em conjunto com a diminuição da flexibilidade (FIDELIS; PATRIZZI; WALSH, 2013). Silva (2017) reforça esse raciocínio afirmando que o idoso sofre modificações em todos os sistemas, afetando a força e a flexibilidade que estão diretamente associadas à melhor função e desempenho das atividades da vida diária.

Em relação a extensão assumida pela problemática do idoso, conforme ressalta Ribeiro (2016), tem provocado mudanças na forma como a sociedade trata e representa a velhice. Assim, pode-se dizer que diferentes imagens da velhice são produzidas em função de determinados meios sociais e conforme os fatores envolvidos. Os significados e valores associados à velhice diferem a partir do 
referencial biológico, fatores não biológicos, como os sociais, econômicos e psicológicos, marcando profundamente o ritmo, a natureza, a estrutura e as significações do que seja envelhecer para o idoso.

O estudo de Jobim e Jobim (2015) afirma que no envelhecimento são grandes as modificações que ocorrem no organismo dos idosos e essas podem ser observadas em todos os aparelhos e sistemas: muscular, ósseo, circulatório, pulmonar, endócrino e imunológico, caracterizando-se pela perda das reservas funcionais dependendo de determinantes genéticos que regulam a longevidade e as condições e estilo de vida. O estudo de Ferreira et al. (2018) complementa a afirmação já citada quando afirma que o envelhecimento, além de alterações biológicas, traz mudanças psicológicas e sociais que afetam o relacionamento do idoso com a família, amigos, sociedade e até consigo mesmo.

O estudo de Matsudo (2010) acrescenta que as alterações fisiológicas que ocorrem durante o envelhecimento podem diminuir a capacidade funcional afetando a saúde e qualidade de vida do idoso.

Para Bittar et al. (2002) um dos principais fatores que limitam a vida do idoso é a falta de equilíbrio. Em $80 \%$ dos casos não possui uma causa específica, mas sim um comprometimento generalizado do sistema de equilíbrio. A maioria dos casos de desequilíbrio acontece entre os 65 e 75 anos, acometendo aproximadamente $30 \%$ dos idosos nessa faixa etária. Ainda segundo Bittar et al. (2002), as quedas são as consequências mais perigosas do desequilíbrio e da dificuldade de locomoção, sendo seguidas por fraturas, deixando os idosos acamados por tempo indeterminado. Em pessoas com mais de 75 anos, são responsáveis por $70 \%$ das mortes acidentais. As manifestações dos distúrbios do equilíbrio corporal têm grande impacto para os idosos, o que pode levá-los à redução de sua autonomia social.

Para Lopes et al. (2010) tanto para situações estáticas e/ou dinâmicas, o equilíbrio consiste em manter o centro de gravidade dentro de uma base de suporte, proporcionando uma maior estabilidade dos segmentos corporais. De natureza 
complexa e multifatorial, a manutenção do equilíbrio do corpo no espaço depende da integração de várias estruturas como a do sistema motor, sensibilidade proprioceptiva, aparelho vestibular, visão, cerebelo dentre outros.

Para McArdle; Katch e Katch (2008) a medida fisiológica do desempenho do indivíduo aumenta durante a maior parte da vida (até os 30 anos) então, há um declínio do rendimento podendo ser de maior conotação sem a prática de exercício físico regular e ainda existe uma grande diferença comparando uma pessoa idosa fisicamente ativa de outra sedentária.

Segundo Papaléo Neto e Carvalho Filho (2002) a estatura começa a diminuir a partir dos 40 anos cerca de $1 \mathrm{~cm}$ por década. Alterações nos discos intervertebrais são responsáveis por esse quadro. Já a pele, com o envelhecimento, as fibras sofrem alteração e a elastina torna-se porosa, perdendo, assim, a elasticidade e dando o aspecto da pele do idoso.

Pereira e Borges (2011) acrescentam que apesar das perdas orgânicas, funcionais e mentais que o idoso adquire, é possível ter um ser humano idoso sadio e com autonomia para realizar as tarefas diárias, manter suas relações intelectuais e sociais com o meio que o rodeia.

\section{TREINAMENTO DE FORÇA E IDOSOS}

Realizar exercícios contra uma determinada resistência é uma das definições de força. Dessa forma, visa gerar uma resposta fisiológica corporal e natural. Esse processo pode ter origem recreativa ou específica, com um objetivo específico a ser atingido (FLECK; KRAEMER, 2017).

Para Dantas (2005) os objetivos de um programa de treinamento resistido não devem limitar-se apenas à melhora da força muscular. O treinamento deve promover procedimentos de treinamento seguro e ser um estímulo para que o participante tenha uma atitude mais positiva sobre o treinamento resistido e a atividade física. 
O exercício físico na terceira idade visa proporcionar com que o idoso enfrente esse novo modo de vida com menos limitações, mais independência e resgatar sua autoestima. A prática regular de atividade física na terceira idade tem se revelado como um fator determinante no que diz respeito à manutenção da qualidade de vida e do bem-estar dos idosos (BORGES; MOREIRA, 2009; MENDONÇA; MOURA; LOPES, 2018).

Nakagava; Rabelo (2007) relatam que é necessário um equilíbrio entre as limitações e as potencialidades de cada indivíduo para que se obtenha um envelhecimento saudável e satisfatório. Dessa forma, o idoso conseguirá passar pelas perdas naturais decorrentes do processo de envelhecimento de forma mais independente e com mais autonomia, melhorando sua qualidade de vida.

O treinamento de força ajuda a diminuir a perda de massa muscular e força associada tipicamente ao envelhecimento. Benefícios adicionais de exercícios regulares incluem o aumento da saúde óssea, redução do risco de osteoporose, aumento da estabilidade da postura, redução de riscos de quedas que podem resultar em fraturas, aumento da flexibilidade e amplitude de movimento (MATSUDO, 2010; FLECK; KRAEMER, 2017).

Segundo Matsudo (2010) o processo de envelhecimento é individual, porém, ao realizar as atividades físicas, pode ser menos impactante, o que proporciona uma melhora na autoestima, das funções cognitivas, diminuição de estresse, além de promover uma interação social, promovendo uma melhora também da saúde mental destes indivíduos. Cassiano (2005) complementa que ao realizar atividades físicas, o indivíduo idoso terá maior independência para atravessar uma rua com segurança, subir no ônibus, fazer compras, realizar visitas sociais e tomar para si a responsabilidade da sua vida econômica, entre outras atividades que venham ampliar a sua rede de convívio social possibilitando, assim, uma melhora da autoestima. 
O treinamento de força na terceira idade pode trazer benefícios tanto físicos, como sociais e psicológicos contribuindo para um estilo de vida mais saudável dos indivíduos que a praticam (MACIEL, 2010).

Santarém (2008) cita alguns efeitos do treinamento de força: aumento da massa muscular, redução da pressão arterial, redução do percentual de gordura, aumento do colesterol (HDL) e ainda funciona como estímulo para a produção hormonal e do sistema imunológico. Desta forma, o exercício atua como excelente fator profilático, prevenindo e melhorando os possíveis fatores de risco para o desenvolvimento de patologias.

$\mathrm{Na}$ terceira idade, o treinamento de força é fundamental, pois ele atua revertendo ou retardando as perdas oriundas do processo natural do envelhecimento, como a perda de massa muscular e óssea, contribuindo dessa forma para uma maior autonomia funcional dos idosos (CRUZ et al., 2008).

Meirelles (2000) destaca como objetivo principal do exercício físico através do treinamento de força na terceira idade, o retardamento do processo inevitável do envelhecimento através da manutenção de um estado suficientemente saudável, senão perfeitamente possível que possibilite a normalização da vida do idoso e afaste os fatores de riscos comuns na terceira idade.

Ainda, segundo Meirelles (2000), à medida que os benefícios do treinamento de força estão relacionados a uma alimentação adequada e balanceada, controle no consumo de bebidas alcoólicas, isenção de hábitos de tabagismo, hora de sono adequada, poder-se-á destacar muitos casos de envelhecimento saudável tanto a nível clínico, como também um desempenho fisiológico.

O estudo de Cruz et al. (2008) explica que o posicionamento da Sociedade Brasileira de Medicina do Esporte (SBME) e Sociedade Brasileira de Geriatria e Gerontologia (SBGG) é que o exercício físico, através do treinamento de força regular, melhora a 
expectativa e qualidade de vida do idoso trazendo inúmeros benefícios principalmente na prevenção e no combate de doenças que podem levar a incapacidades.

Vários são os benefícios para os seres humanos que optam por um estilo de vida ativo através de exercícios físicos orientados por um profissional: melhoria na capacidade cardiorrespiratória; melhora no equilíbrio; melhoria no andar; manutenção e/ou aumento da densidade óssea; controle do diabetes, artrite e doenças cardíacas; melhoria na ingestão de alimentos; diminuição da depressão; fortalecimento geral dos músculos do corpo; melhoria nos reflexos; melhoria na sinergia motora das reações posturais; melhoria da flexibilidade e mobilidade; manutenção do peso corporal; diminuição das lesões musculares; melhoria da autoestima, entre outros (TAKAHASHI, 2004).

Penna e Santo (2006) destacam a importância de uma avaliação médica cuidadosa para o idoso que vai iniciar a prática de exercícios físicos. Dessa forma, toda prescrição será feita baseada nas individualidades de cada idoso, levando em consideração suas possíveis limitações e possibilidades. Esse programa deve levar em consideração as alterações ocorridas durante o processo de envelhecimento, proporcionando melhorias nas capacidades motoras envolvidas na realização das atividades do cotidiano e favorecendo a capacidade de trabalho e de lazer bem como alterando a taxa de declínio do estado funcional, visando à melhoria da qualidade de vida do idoso.

Segundo os estudos de Takahashi (2004), através dos exercícios, além de combater a obesidade, evita e retarda o surgimento de diabetes, melhora a capacidade aeróbica, também é possível aumentar a massa óssea ou reduzir sua perda (osteoporose), diminui os riscos de quedas e de fraturas de fêmur e de quadril. Além disso, o fortalecimento muscular reduz dores já existentes provenientes de doenças como artrite, tendinite, bursite, artrose e problemas de coluna.

Cruz et al. (2008) elucida que as opções de exercícios que se propõem para os idosos não diferenciam em demasia daqueles que atualmente são praticados nas academias. 
A linha de atuação do treinamento de força que deverão direcionar a escolha do exercício mais indicado, contendo variáveis de cargas, repetições, entre outros, individualizados e modalidades que proporcionem ao idoso maior satisfação para realizá-lo. Estas atividades precisam atender vários aspectos que proporcionem saúde globalizada ao idoso, como uma simples manipulação de um objeto, a um incremento favorável na estrutura biopsicofisiológica.

Ferreira (2011) cita que o treinamento de força promove uma infinidade de benefícios para o praticante, entre eles: prevenção e tratamento da osteoporose, manutenção ou aumento da densidade mineral óssea, prevenção da perda de massa óssea, aumento de força e da massa muscular, aumento da flexibilidade, aumento do VO2 máx., controle da glicemia, melhora do perfil lipídico, redução do peso corporal, melhora do equilíbrio e da marcha, menor dependência para a realização das atividades diárias, melhora da autoestima e da autoconfiança, diminuição da incidência de quedas, diminuição do risco de fraturas, dentre outros.

\section{FLEXIBILIDADE}

Segundo Roberts e Robergs (2002) a flexibilidade é a capacidade de elevar ao máximo a intensidade articular no movimento, além disso, é considerada como um dos componentes da aptidão física relacionada à saúde e também à performance, é específica das articulações podendo ser aperfeiçoada com a prática.

Para Dantas et al. (2009) a flexibilidade promoverá aumento da capacidade de mobilidade articular, influenciando aspectos como o controle ou eliminação da tensão muscular, das substâncias tóxicas produzidas durante a atividade física e da rigidez articular, aperfeiçoamento motor, eficiência mecânica, profilaxia de lesões, consciência corporal entre outros.

Para Dantas (2005) existem quatro fatores responsáveis pelo grau de flexibilidade de uma articulação: mobilidade, elasticidade, plasticidade e maleabilidade. As principais 
limitações são da própria articulação (limite de mobilidade articular) e a musculatura a ela associada.

Dantas (2005) ainda explica que as reivindicações de bons níveis de flexibilidade em relação à saúde sejam amplamente descritas, embora não se tenha conseguido estabelecer de forma científica, quanto de flexibilidade seria necessário para o ser humano, ela é responsável pela qualidade física da execução de movimentos voluntários em amplitudes máximas (respeitando os limites morfológicos). A flexibilidade é dependente da elasticidade muscular e da mobilidade articular e é necessária para uma perfeita execução de atividades físicas.

O estudo de Silva (2017) acrescenta que a flexibilidade é percebida como um componente relevante da aptidão física, incluído à saúde e ao desempenho do indivíduo, fazendo-se necessário para a realização de atividades de vida diária com uma boa qualidade de vida.

Sobre a flexibilidade, autores como Weineck (2003), Santarém (2011) e Fleck e Kraemer (2017) explicam que ela pode ser classificada em geral ou específica, ativa ou passiva, estática ou dinâmica como segue:

- A flexibilidade geral pode ser observada nos movimentos que englobam todas as articulações. Os principais e grandes sistemas articulares são exigidos na flexibilidade geral, como ombros, coluna vertebral e quadris.

- A flexibilidade específica, como o próprio nome diz, refere-se ao grau de amplitude de movimento de articulações específicas.

- Quando uma articulação se movimenta até sua amplitude máxima devido a contração dos músculos agonistas e, consequentemente, pelo relaxamento dos antagonistas, temos a flexibilidade ativa. Ela é obtida sem qualquer tipo de auxílio externo (suporte de um parceiro ou de aparelhos) ao praticante.

- Flexibilidade passiva utiliza o auxílio de forças externas, buscando uma maior ADM através dessas forças externas. 
- A flexibilidade estática é conseguida quando o praticante vai até o ponto de maior ADM de forma lenta e gradual. Em seguida, essa posição é sustentada durante o tempo indicado pelo profissional da área.

- A flexibilidade dinâmica é obtida pela contração voluntária dos músculos motores, buscando grande ADM e podendo ser feita com velocidade rápida ou mais lenta e controlada.

O estudo de Nahas (2013) acrescenta que o treinamento de flexibilidade para idosos deve ser diferenciado com relação a outras faixas etárias. Esse treinamento com idosos, devido ao aumento das tensões sobre os ossos, deverá ser realizado com cautela e adaptação prévia aos movimentos a serem trabalhados a fim de evitar lesões ou desconforto.

\section{EFEITOS DO TREINAMENTO DE FORÇA NA FLEXIBILIDADE DOS IDOSOS}

Observa-se que a flexibilidade é diretamente relacionada à saúde, pois tem íntima relação com a capacidade de executar tarefas da vida diária e à prevenção de doenças, sendo, portanto, um componente da aptidão física importante para a manutenção de bons níveis de saúde e qualidade de vida, pois a flexibilidade diminuída restringe as possibilidades de movimento, além de aumentar as chances de lesões articulares e musculares (SILVA, 2017; MOURA et al., 2018).

De acordo com Dantas (2009), existem muitos efeitos da prática do exercício físico, dentre eles, o aumento da força através do treinamento de força, sendo o mais indicado para sistema neuromuscular, aperfeiçoando a coordenação nos aspectos de recrutamento de unidades motoras, que biomecanicamente pode-se dizer que a qualidade de vida depende essencialmente de força e de flexibilidade.

McArdle; Katch e Katch (1998) afirmam que para os idosos as adaptações ocorridas com o treinamento de força acontecem tanto em homens quanto em mulheres, sem a influência do envelhecimento.

RC: 106653

Disponível em: https://www.nucleodoconhecimento.com.br/educacao-fisica/efeitosdo-treinamento 
O estudo de Moura et al. (2018), por meio de dados significativos obtidos nos treinamentos realizados, constata-se que a melhora ou manutenção da força muscular e flexibilidade articular tem grande influência no equilíbrio corporal em idosos, reduzindo o risco de quedas e melhorando a qualidade de vida. Dessa forma, a independência funcional é prolongada, fato que é muito importante para os idosos.

Albino et al. (2012) esclarece que, no envelhecimento, o exercício através do treinamento de força pode modificar e aperfeiçoar as funções de alguns tecidos importantes para a melhoria da flexibilidade como o tecido muscular, o tecido ósseo e as estruturas do tecido conjuntivo. O estudo de Souza et al (2015) complementa ressaltando que a prática regular de exercício físico através do treinamento de força poderá melhorar outros fatores essenciais como a diminuição das dores articulares e a melhoria do padrão de recrutamento motor, amparando assim, de forma expressiva na melhora e desenvolvimento da flexibilidade na terceira idade.

O estudo de Silva (2017) explana que a flexibilidade é uma variável essencial da aptidão física relacionada à saúde, possuindo uma íntima relação com a estabilidade corporal, a mobilidade de vários segmentos corporais e articulares, bem como uma estreita relação com a manutenção de postura. Assim, a manutenção dos níveis de flexibilidade em uma dimensão para as práticas exigidas no cotidiano é de extrema importância para a manutenção da qualidade de vida do idoso.

\section{CONSIDERAÇÕES FINAIS}

Ao concluir este estudo, observou-se que a população idosa tem aumentado consideravelmente em todo o mundo e esse fato tem despertado a atenção de diversas área do conhecimento a fim de elaborar políticas públicas direcionadas para o envelhecimento saudável dessa população, pois o processo de envelhecimento é acompanhado, por um estilo de vida inativo, favorecendo ao declínio das capacidades físicas, o que gera perdas na aptidão funcional e consequente dependência, ocorrendo grandes modificações no organismo dos idosos. 
O treinamento de força em idosos é fundamental, pois ele atua revertendo ou retardando as perdas oriundas do processo natural do envelhecimento, como a perda de massa muscular e óssea, contribuindo dessa forma para uma maior autonomia funcional dos idosos. A flexibilidade em idosos é um componente importante da aptidão física, garantindo uma maior amplitude nos movimentos corporais, promovendo melhorias na execução de atividades cotidianas com efeitos benéficos na qualidade de vida, o aumento da densidade óssea e muscular, estimula a proliferação do tecido conjuntivo elástico nos músculos, tendões, ligamentos e cápsula articular.

Observou-se que ambas as capacidades físicas, força e flexibilidade, estão diretamente relacionadas a qualidade de vida dos idosos e permitem uma maior autonomia e independência nas suas atividades diárias. Mas seria o treinamento de força em idosos capaz de melhorar também a flexibilidade desse público? Segundo a literatura consultada, pôde-se observar que com o treinamento de força, os idosos adquiriram aumento de força, resistência e na flexibilidade, o que gera um ganho no desempenho funcional, melhora da qualidade vida, retardando alguns efeitos fisiológicos do envelhecimento.

Deste modo, cabe aos profissionais de educação física utilizar os conhecimentos adquiridos tanto teóricos como práticos como forma de prescrever a atividade de maneira correta e segura possibilitando o idoso envelhecer com saúde e melhor qualidade de vida.

\section{REFERÊNCIAS}

ALBINO, I. L. R.; FREITAS, C. I. R.; TEIXEIRA, A. R.; GONÇALVES, A. K.; SANTOS, A. M. P. V.; BOS, Â. J. G. Influência do treinamento de força muscular e de flexibilidade articular sobre o equilíbrio corporal em idosas. Revista Brasileira de Geriatria e Gerontologia. Rio de Janeiro, v. 15, n. 1, p. 17-25, 2012. 
BITTAR, R. S. M.; PEDALINI, M. E. B.; BOTTINO, M. A.; FORMIGONI, L. G. Síndrome do desequilíbrio no idoso. Pró-fono, Revista de Atualização Científica, v. 14, n. 1, p. 119-28. 2002.

BORGES, M.; MOREIRA, A. K. Influências da prática de atividades físicas na terceira idade: estudo comparativo dos níveis de autonomia para o desempenho nas AVDs e AIVDs entre idosos ativos fisicamente e idosos sedentários. Motriz, Rio Claro, v.15 n.3 p.562-573, jul./set. 2009.

CASSIANO, J. G. Promovendo saúde e qualidade de vida em adultos maduros e idosos. Anais. 8 encontro de extensão da UFMG. Belo Horizonte. De 03 a 08 de outubro de 2005.

CIOSAK, S. I.; BRAZ, E.; COSTA, M. F. B. N. A.; NAKANO, N. G. R.; RODRIGUES, J.; ALENCAR, R. A.; ROCHA, A. C. A. L. Senescência e senilidade: novo paradigma na Atenção Básica de Saúde. Rev Esc Enferm USP. V.45, n. 2, p :1763-8, 2011.

CRUZ, I. R. D. ; SOUZA, D. S. ; LESSA, K. B. Q. ; NAGEM, M. P. ; DURÃES, G. M. ; LESSA JUNIOR, A. Nível de Atividade Física Desportiva de Acadêmicos do Curso de Educação Física da Funorte. In: Jornada Nacional de Atividade Física, Esporte e Saúde, Montes Claros. Jornada Nacional de Atividade Física, Esporte e Saúde. 2008.

DANTAS, E. H. M. Flexibilidade. Alongamento e flexionamento. 6. ed. Rio de Janeiro: Shape, 2005.

DANTAS, E. H. M.; BEZERRA, J. C. P.; MELLO, D. B. Fitness Saúde e Qualidade de Vida. In: VIANNA, J.; NOVAES, J. Personal Training \& Condicionamento Físico em Academia. 3 Ed. Rio de Janeiro: Shape, pp.297- 335, 2009

FERREIRA, L. K.; MEIRELES, J. F. F.; FERREIRA, M. E. C. Avaliação do estilo e qualidade de vida em idosos: uma revisão de literatura. Revista Brasileira de Geriatria e Gerontologia. 21 (05). Rio de Janeiro. 2018. 
FIDELIS, L.T.; PATRIZZI, L.J.; WALSH, I.A.P. Influência da prática de exercícios físicos sobre a flexibilidade, força muscular manual e mobilidade funcional em idosos. Revista Brasileira de Geriatria e Gerontologia. Rio de Janeiro. 2013 p. 109-1

FLECK, S. J.; KRAEMER, W. J. Fundamentos do treinamento de força muscular. 3. ed. São Paulo /SP: Editora Artmed, 2017.

GONÇALVES, R.; GURJÃO, A. L. D.; GOBBI, S. Efeitos de oito semanas no treinamento de força na flexibilidade de idosos. Rev. Bras. Cineantropom. Desempenho Hum. 2007; 9(2)145-153

JARDIM, V. C. F. da S. Perfil epidemiológico e grau de autonomia de mulheres idosas participantes de grupos de convivência, no município de Olinda - PE. 2007. 108 f. Dissertação (Mestrado em Saúde Pública) - Centro de Pesquisas Aggeu Magalhães, Fundação Oswaldo Cruz, Recife, 2007.

JOBIM, F. A. R. C.; JOBIM, E. F. C. Atividade física, nutrição e estilo de vida no envelhecimento. UNOPAR Cient Ciênc Biol Saúde 2015; 17(4):298-308

LOCKS, R. R. et al. Efeitos do treinamento de força e flexibilidade no desempenho funcional de idosos saudáveis. Rev. bras. fisioter. vol.16, n.3, pp.184-190. 2012.

LOPES, M. N. S. S.; PASSERINI, C. G.; TRAVENSOLO, C. F. Eficácia de um protocolo fisioterapêutico para equilíbrio em idosos institucionalizados. Ciências Biológicas e da Saúde. v.31, n. 2. 2010.

MACIEL, M. G. Atividade física e funcionalidade do idoso. Motriz, Rio Claro, v.16 n.4, p.1024-1032, out./dez. 2010.

MATSUDO, S. M. M. Avaliação do idoso Física e Funcional. 3. ed. Santo André: Gráfica Mali, 2010. 
McARDLE D. W.; KATCH I. F.e KATCH L. K Victor. Fisiologia do exercício, energia nutrição e desempenho humano. 4. ed. São Paulo - SP: Editora Guanabara Koogan S.A., 1998.

MEIRELLES, Morgana E. A. Atividade física na 3 Idade. 2. ed. Rio de Janeiro - RJ: Sprint, 2000.

MENDONÇA, C. S.; MOURA, S. K. M. S. F.; LOPES, D. T. Benefícios do treinamento de força para idosos: Revisão Bibliográfica. Revista Campo do Saber. v. 4, n. jan/jun de 2018.

MIRANDA, G. M. D.; MENDES, A. C. G.; SILVA, A. L. A. O envelhecimento populacional brasileiro: desafios e consequências sociais atuais e futuras. Rev. bras. geriatr. gerontol. vol.19 no.3 Rio de Janeiro May/June 2016

MOURA, D. P.; TONON, D. R.; NASCIMENTO, D. F. Efeito agudo do treinamento de força sobre a flexibilidade de membros inferiores. Revista Brasileira de Prescrição e Fisiologia do Exercício, São Paulo. v.12. n.72. p.96-100. Jan./Fev. 2018.

MOURA, E. S.; CASTRO, H. O.; AGUIAR, S. S.; BORGES JÚNIOR, M.; COSTA, G. C. T.; PIRES, F. O.; SOARES, K. V. B. C. Avaliação do nível de força e flexibilidade de idosos praticantes de atividades físicas. Revista Brasileira de Prescrição e Fisiologia do Exercício, São Paulo. v.12. n.75. Suplementar 1. p.496-502. Jan./Jun. 2018.

NAHAS, M. V. Atividade física, saúde e qualidade de vida: conceitos e sugestões para um estilo de vida ativo. 6. ed. Londrina (PR): Midiograf, 2013.

NAKAGAVA, B. K. C.; RABELO, R. J. Perfil da qualidade de vida de mulheres idosas praticantes de hidroginástica. MOVIMENTUM - Revista Digital de Educação Física. Ipatinga: Unileste-MG, v. 2, n.1, Fev./jul., 2007. 
NASCIMENTO, C. M. C.; AYAN, C.; CANCELA, J. M.; PEREIRA, J. R.; ANDRADE, L. P.; GARUFFI, M.;GOBBI, S.; STELLA, F. Exercícios físicos generalizados capacidade funcional e sintomas depressivos em idosos brasileiros. Rev Bras Cineantropom Desempenho Hum. v.15, n. 4, '. 486-497, 2013.

PAPALÉO NETO, M., CARVALHO FILHO, E. Geriatria: fundamentos, clínica e terapêutica. 2. ed. São Paulo: Atheneu, 2002.

PENNA, F. B.; SANTO, F. H. E. O movimento das emoções na vida dos idosos: um estudo com um grupo da terceira idade. Revista Eletrônica de Enfermagem. São Paulo, v.8, n. 1, p. 17-24, 2006.

PEREIRA, E. S.; BORGES, Í. S. Análise da percepção geral de saúde e da qualidade de vida de idosos praticantes de hidroginástica a partir do instrumento SF-36. Revista Brasileira de Prescrição e Fisiologia do Exercício, São Paulo, v.5, n.27, p.278-284. Maio/Junho. 2011.

RIBEIRO, G. J. M.; HAGALE, M. M.; VASCONCELOS, A. P. S. L. Efeitos do treinamento resistido sobre a força em indivíduos idosos. Revista Eletrônica da Faculdade Metodista Granbery Curso de Educação Física. N. 20, JAN/JUN 2016.

ROBERGS, R. A.; ROBERTS, S. O. Princípios fundamentais de Fisiologia do Exercício para aptidão, desempenho e saúde. São Paulo: Phorte, 2002.

SANTARÉM, J. M. Promoção da saúde dos idosos: a importância da atividade física. 2011. Disponível em: http://www.saudetotal.com/santarem.htm. Acesso em 10 de maio de 2020.

SANTARÉM, J. M. Fisiologia do Exercício e Treinamento Resistido a Saúde, na Doença e no Envelhecimento. 2008. http://www.culturismo.com.br/index/asp http://saudetotal.com/cecafi/texto.htm Acesso 18 de outubro de 2018. 
SHEPHARD. R.J. Envelhecimento, atividade física e saúde. São Paulo: Phorte, 2003.

SILVA, J. V. L. Benefícios do treinamento resistido em idosos: Uma revisão da literatura. Vitória de Santo Antão, 2017. 27 f. TCC (Graduação em Educação Física) - Universidade Federal de Pernambuco, CAV, Bacharelado em Educação Física 2017.

SOUZA, W. C.; MASCARENHAS, L. P. G.; GRZELCZAL, M. T. TAJES, D.; BRASILINO, F. F.; LIMA, V. A. Exercício físico na promoção da saúde na terceira idade. Saúde Meio Ambient. v. 4, n. 1, p. 55-65, jan./jun. 2015

TAKAHASHI, S. R. S. Benefícios da atividade física na melhor idade. Revista Digital - Buenos Aires - Ano 10 - N 74 - Julho de 2004.

WEINECK, J. Treinamento Ideal: Instrução técnica sobre o Desempenho Fisiológico, incluindo considerações específicas de treinamento infantil e juvenil. 9. ed. São Paulo: Manole, 2003.

Enviado: Junho, 2021.

Aprovado: Fevereiro, 2022. 